Supporting information for

\title{
Hybrid Charge-Transfer Semiconductors: $\left(\mathrm{C}_{7} \mathrm{H}_{7}\right) \mathrm{SbI}_{4},\left(\mathrm{C}_{7} \mathrm{H}_{7}\right) \mathrm{BiI}_{4}$, and Their Halide Congeners
}

Iain W. H. Oswald, Eve M. Mozur, Ian P. Moseley, Hyochul Ahn, and James R. Neilson* *corresponding author: J. R. N. (James.Neilson@ colostate.edu)

Department of Chemistry, Colorado State University, Fort Collins, CO, 80523-1872, United States 


\section{Molar solubility studies}

Molar solubilities were determined by adding $0.100 \mathrm{~mL}$ aliquots of the corresponding hydrohalic acid (HI was stabilized) to a vial containing $\sim 0.050 \mathrm{~g}$ of the microcrystalline product until completely dissolved under stirring.

Table S1. Molar Solubilities (mol/L) of $\left(\mathrm{C}_{7} \mathrm{H}_{7}\right) \mathrm{MX}_{4}$ in $\mathrm{HX}(a q)$.

\begin{tabular}{ll}
\hline Compound & Molar Solubility (M) \\
$\left(\mathrm{C}_{7} \mathrm{H}_{7}\right) \mathrm{SbCl}_{4}$ & 0.0525 \\
$\left(\mathrm{C}_{7} \mathrm{H}_{7}\right) \mathrm{SbBr}_{4}$ & 0.0280 \\
$\left(\mathrm{C}_{7} \mathrm{H}_{7}\right) \mathrm{SbI}_{4}$ & 0.0085 \\
$\left(\mathrm{C}_{7} \mathrm{H}_{7}\right) \mathrm{BiCl}_{4}$ & 0.1276 \\
$\left(\mathrm{C}_{7} \mathrm{H}_{7}\right) \mathrm{BiBr}_{4}$ & 0.0381 \\
$\left(\mathrm{C}_{7} \mathrm{H}_{7}\right) \mathrm{BiI}_{4}$ & 0.0136 \\
\hline
\end{tabular}

Thermal Gravimetric Analysis curves.
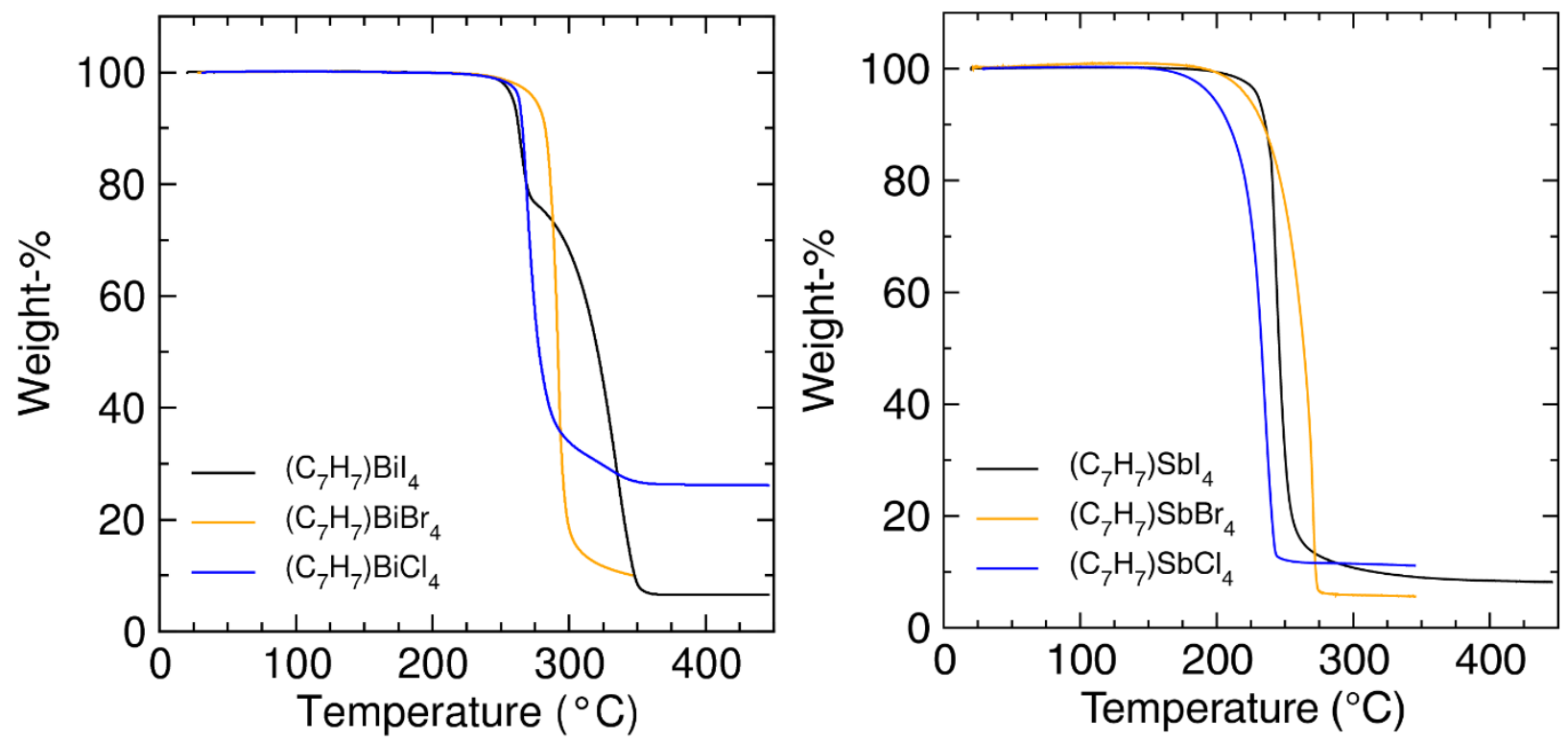

Figure S1. Thermal decomposition curves for all compounds studied. 

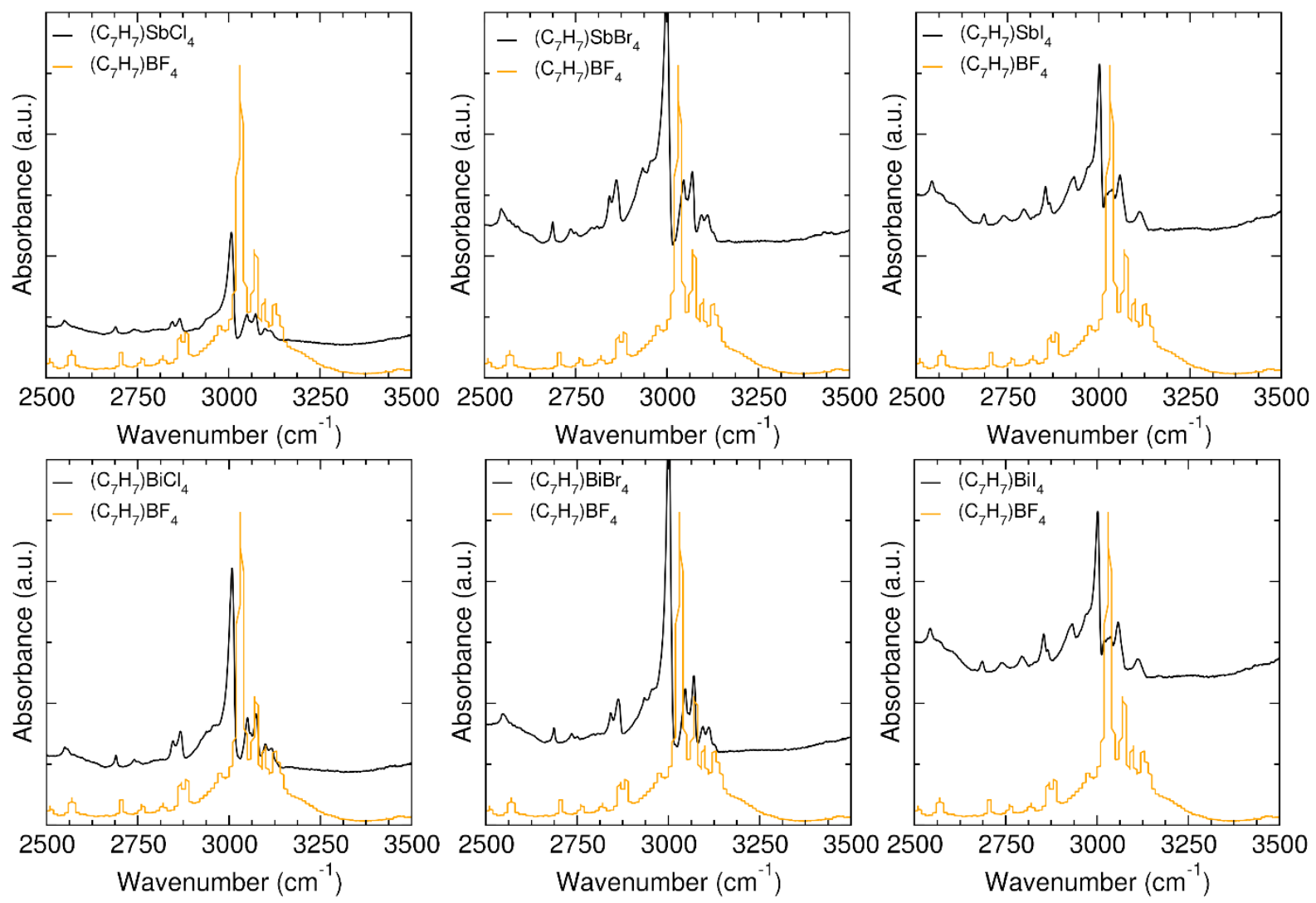

Figure S2. ATR-FTIR spectra of all compounds studied and the tropylium tetrafluoroborate precursor. The strongest band around $3060 \mathrm{~cm}^{-1}$ corresponds to the $\mathrm{C}-\mathrm{H}$ stretch.

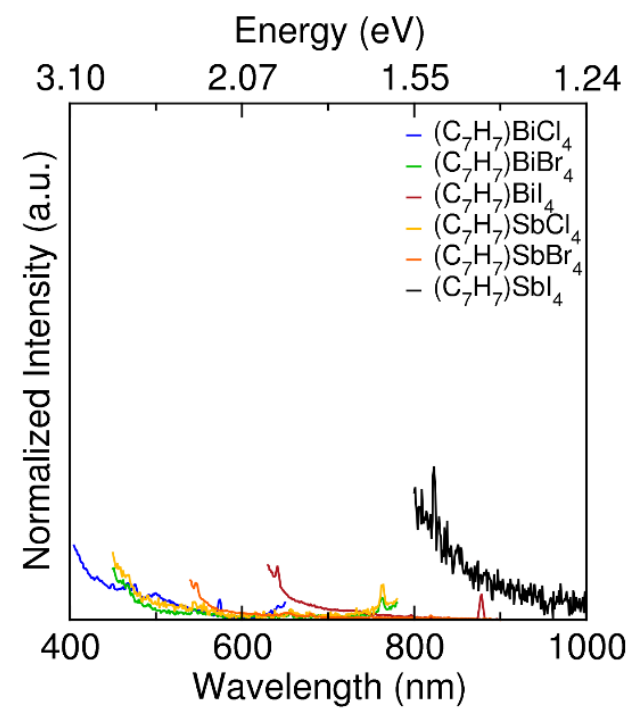

Figure S3. Photoluminescence spectra showing no appreciable signal for all samples at room temperature. Higher intensities for all samples at lower wavelengths are from the incident light source. 
Table S2. $\left(\mathrm{C}_{7} \mathrm{H}_{7}\right) \mathrm{MX}_{4}\left(\mathrm{M}=\mathrm{Sb}^{3+}, \mathrm{Bi}=; \mathrm{X}=\mathrm{Cl}^{-}, \mathrm{Br}^{-}, \mathrm{I}^{-}\right)$Atomic Positions and Thermal Parameters for models obtained from Rietveld refinement.

\begin{tabular}{|c|c|c|c|c|}
\hline Compound/Atom & $x$ & $y$ & $z$ & $\mathrm{~B}_{i s o} * / \mathrm{B}_{e q}$ \\
\hline \multicolumn{5}{|l|}{$\left(\mathrm{C}_{7} \mathrm{H}_{7}\right) \mathrm{SbCl}_{4}$} \\
\hline $\mathrm{Sb} 1$ & 0.5 & $0.5842(3)$ & 0.25 & $6.5(3)$ \\
\hline $\mathrm{Cl1}$ & $0.6252(85)$ & $0.5891(6)$ & $0.6699(17)$ & $5.8(4)$ \\
\hline $\mathrm{Cl} 2$ & $0.6154(80)$ & $0.7064(6)$ & $0.2193(15)$ & $8.8(9)$ \\
\hline $\mathrm{C} 1$ & 0.5 & 0.952 & 0.25 & 10 \\
\hline $\mathrm{H} 1$ & 0.5 & 0.888539 & 0.250004 & 9.395863 \\
\hline $\mathrm{C} 2$ & 0.59 & 0.985 & 0.293 & 6.395504 \\
\hline $\mathrm{H} 2$ & 0.645543 & 0.943602 & 0.333852 & 7.73777 \\
\hline $\mathrm{C} 3$ & 0.614 & 1.074 & 0.285 & 3.20415 \\
\hline H3 & 0.67959 & 1.082955 & 0.290786 & 9.711691 \\
\hline $\mathrm{C} 4$ & 0.438 & 1.149 & 0.228 & 7.579856 \\
\hline $\mathrm{H} 4$ & 0.403932 & 1.205149 & 0.211782 & 9.080036 \\
\hline \multicolumn{5}{|l|}{$\left(\mathrm{C}_{7} \mathrm{H}_{7}\right) \mathrm{SbBr}_{4}$} \\
\hline $\mathrm{Sb} 1$ & 0.5 & $0.4164(3)$ & 0.75 & $4.31(16)$ \\
\hline $\mathrm{Br} 1$ & $0.3644(4)$ & $0.4158(3)$ & $0.3134(7)$ & $4.15(17)$ \\
\hline $\mathrm{Br} 2$ & $0.3644(4)$ & $0.2901(3)$ & $0.7633(7)$ & $3.98(16)$ \\
\hline $\mathrm{C} 1$ & 0.5 & 0.031324 & 1.75 & 12.80988 \\
\hline H1 & 0.499999 & 0.112269 & 1.749999 & 8.922122 \\
\hline $\mathrm{C} 2$ & 0.409113 & 0.004248 & 1.724361 & 7.421943 \\
\hline $\mathrm{H} 2$ & 0.356496 & 0.054767 & 1.689099 & 8.922122 \\
\hline $\mathrm{C} 3$ & 0.382049 & -0.080667 & 1.711227 & 6.906772 \\
\hline H3 & 0.316214 & -0.086969 & 1.683519 & 8.922122 \\
\hline $\mathrm{C} 4$ & 0.445341 & -0.153894 & 1.719754 & 5.046918 \\
\hline $\mathrm{H} 4$ & 0.41356 & -0.208294 & 1.724247 & 8.922122 \\
\hline \multicolumn{5}{|l|}{$\left(\mathbf{C}_{7} \mathbf{H}_{7}\right) \mathrm{SbI}_{4}$} \\
\hline $\mathrm{Sb} 1$ & 0.5 & $0.5837(6)$ & 0.25 & $5.5(4)$ \\
\hline I1 & $0.6382(5)$ & $0.5818(4)$ & $0.6933(9)$ & $6.1(4)$ \\
\hline I2 & $0.6384(5)$ & $0.7165(4)$ & $0.2184(9)$ & $6.5(4)$ \\
\hline $\mathrm{C} 1$ & 0.5 & 0.953146 & 0.25 & 4.581434 \\
\hline H1 & 0.5 & 0.888539 & 0.250004 & 9.395863 \\
\hline $\mathrm{C} 2$ & 0.587583 & 0.982437 & 0.279263 & 8.639739 \\
\hline $\mathrm{H} 2$ & 0.645543 & 0.943602 & 0.333852 & 7.73777 \\
\hline $\mathrm{C} 3$ & 0.608297 & 1.083103 & 0.269362 & 8.132554 \\
\hline $\mathrm{H} 3$ & 0.67959 & 1.082955 & 0.290786 & 9.711691 \\
\hline
\end{tabular}




\begin{tabular}{|c|c|c|c|c|}
\hline $\mathrm{C} 4$ & 0.437750 & 1.139709 & 0.245103 & 7.579856 \\
\hline $\mathrm{H} 4$ & 0.403932 & 1.205149 & 0.211782 & 9.080036 \\
\hline \multicolumn{5}{|c|}{$\left(\mathrm{C}_{7} \mathrm{H}_{7}\right) \mathrm{BiCl}_{4}$} \\
\hline Bi1 & 0.5 & $0.4184(2)$ & 0.75 & $6.0(2)$ \\
\hline $\mathrm{Cl} 1$ & $0.3743(7)$ & $0.4242(9)$ & $0.3319(14)$ & $6.5(4)$ \\
\hline $\mathrm{Cl} 2$ & $0.3668(8)$ & $0.2898(8)$ & $0.7730(15)$ & $9.5(5)$ \\
\hline $\mathrm{C} 1$ & 0.5 & 0.054189 & 1.75 & 2.707936 \\
\hline $\mathrm{H} 1$ & 0.499999 & 0.112269 & 1.749999 & 8.922122 \\
\hline $\mathrm{C} 2$ & 0.398575 & 0.001049 & 1.719612 & 7.421943 \\
\hline $\mathrm{H} 2$ & 0.356496 & 0.054767 & 1.689099 & 8.922122 \\
\hline $\mathrm{C} 3$ & 0.380787 & -0.080243 & 1.714553 & 6.877442 \\
\hline H3 & 0.316214 & -0.086969 & 1.683519 & 8.922122 \\
\hline $\mathrm{C} 4$ & 0.43 & -0.162951 & 1.75 & 1.062935 \\
\hline $\mathrm{H} 4$ & 0.41356 & -0.208294 & 1.724247 & 8.922122 \\
\hline \multicolumn{5}{|c|}{$\left(\mathrm{C}_{7} \mathrm{H}_{7}\right) \mathrm{BiBr}_{4}$} \\
\hline Bi1 & 0.5 & $0.4132(3)$ & 0.75 & $3.7(2)$ \\
\hline $\mathrm{Br} 1$ & $0.3628(5)$ & $0.4228(4)$ & $0.3103(8)$ & $5.3(3)$ \\
\hline $\mathrm{Br} 2$ & $0.3622(5)$ & $0.2853(4)$ & $0.7772(10)$ & $5.9(3)$ \\
\hline $\mathrm{C} 1$ & 0.5 & 0.0486 & 1.75 & 12.80988 \\
\hline $\mathrm{H} 1$ & 0.499999 & 0.112269 & 1.749999 & 8.922122 \\
\hline $\mathrm{C} 2$ & 0.4104 & 0.0133 & 1.714 & 7.421943 \\
\hline $\mathrm{H} 2$ & 0.356496 & 0.054767 & 1.689099 & 8.922122 \\
\hline $\mathrm{C} 3$ & 0.3849 & -0.0756 & 1.708 & 6.906772 \\
\hline $\mathrm{H} 3$ & 0.316214 & -0.086969 & 1.683519 & 8.922122 \\
\hline $\mathrm{C} 4$ & 0.4449 & -0.1517 & 1.732 & 5.046918 \\
\hline $\mathrm{H} 4$ & 0.41356 & -0.208294 & 1.724247 & 8.922122 \\
\hline
\end{tabular}

Table S3. $\left(\mathrm{C}_{7} \mathrm{H}_{7}\right) \mathrm{BiI}_{4}$ Atomic Positions and Thermal Parameters obtained from single crystal diffraction.

\begin{tabular}{lllll}
\hline Compound/Atom & $\boldsymbol{x}$ & $\boldsymbol{y}$ & $\boldsymbol{z}$ & $U_{\text {iso }} * U_{\mathrm{eq}}$ \\
\hline$\left(\mathbf{C}_{7} \mathbf{H}_{7}\right) \mathbf{B i I}_{4}$ & & & & \\
$\mathrm{Bi} 1$ & 0.500000 & $0.41367(3)$ & 0.750000 & $0.03235(13)$ \\
$\mathrm{I} 1$ & $0.36241(4)$ & $0.41953(3)$ & $0.30499(7)$ & $0.03760(15)$ \\
$\mathrm{I} 2$ & $0.28047(4)$ & $0.77924(9)$ & $0.04769(17)$ \\
$\mathrm{C} 1$ & $0.35728(4)$ & $0.0486(16)$ & 1.750000 & $0.094(2)$ \\
$\mathrm{H} 1$ & 0.500000 & 0.112269 & 1.749999 & $0.113^{*}$ \\
$\mathrm{C} 2$ & 0.499999 & $0.0133(11)$ & $1.714(2)$ & $0.094(2)$
\end{tabular}




\begin{tabular}{lllll}
$\mathrm{H} 2$ & 0.356496 & 0.054767 & 1.689099 & $0.113^{*}$ \\
$\mathrm{C} 3$ & $0.3849(14)$ & $-0.0756(9)$ & $1.708(2)$ & $0.094(2)$ \\
$\mathrm{H} 3$ & 0.316214 & -0.086969 & 1.683519 & $0.113^{*}$ \\
$\mathrm{C} 4$ & $0.4449(11)$ & $-0.1517(10)$ & $1.732(2)$ & $0.094(2)$ \\
$\mathrm{H} 4$ & 0.413560 & -0.208294 & 1.724247 & $0.113^{*}$ \\
\hline
\end{tabular}

${ }^{a} U_{\text {eq }}$ is defined as one-third of the trace of the orthogonalized $U_{\mathrm{ij}}$ tensor.

Table S4. Structural descriptions in the VASP POSCAR format of all compounds after relaxation of DFT-calculated forces.

\begin{tabular}{|c|c|c|}
\hline C7H7SbCl4 & & \\
\hline 1.00000000000000 & & \\
\hline 7.2613462355444129 & 0.0115352679251104 & 0.0135582920670977 \\
\hline 3.5472773158399322 & 8.8024479635463830 & 0.0724580579730636 \\
\hline 3.5473783186343941 & -1.2922192198982441 & 8.7075093278437627 \\
\hline $\begin{array}{lllll}\mathrm{Sb} & \mathrm{Cl} & \mathrm{C} & \mathrm{H}\end{array}$ & & \\
\hline $\begin{array}{llll}2 & 8 & 14 & 14\end{array}$ & & \\
\hline Direct & & \\
\hline 0.2499923380877931 & 0.42476601662875880 & 5752395647413593 \\
\hline 0.7500076619122069 & 0.57523398337124120 & 4247604352586407 \\
\hline 0.6794783905665227 & 0.28628304540556120 & 4385369020158194 \\
\hline 0.3205216094334773 & 0.71371695459443880 & 5614630679841781 \\
\hline 0.1795079937336084 & 0.43850283750042960 & 2862625823911173 \\
\hline 0.8204920362663870 & 0.56149713249956790 & 7137374176088827 \\
\hline 0.2291334016049973 & 0.17269375378705120 & 5761598398901668 \\
\hline 0.7708665683950002 & 0.82730624621294880 & 4238401601098332 \\
\hline 0.7291821169684951 & 0.57614412390638140 & 1726758756284497 \\
\hline 0.2708178830315049 & 0.42385587609361860 & 8273241243715503 \\
\hline 0.2500178547128939 & $\begin{array}{lll}0.0632018920899711 & 0\end{array}$ & 9367895901727010 \\
\hline 0.7499821452871061 & 0.93679812991003080 & 0632104098272990 \\
\hline 0.7057343392594504 & 0.08507577918414460 & 1208105961171100 \\
\hline 0.2942656607405496 & 0.91492422081585540 & 8791894338828925 \\
\hline 0.2057456389066061 & 0.12080280264400050 & 0850774025982659 \\
\hline 0.7942543760933916 & 0.87919722035599790 & 9149225974017341 \\
\hline 0.6999149613114781 & 0.21209637573308270 & 0431889912038415 \\
\hline 0.3000850386885290 & 0.78790360926691960 & 9568109867961567 \\
\hline 0.1998984539135833 & 0.04320651242257160 & 2120839030602113 \\
\hline 0.8001015610864144 & 0.95679346557742660 & 7879160819397910 \\
\hline 0.2705382526105140 & 0.77719308571994360 & 1100479512158472 \\
\hline 0.7294617473894860 & 0.22280691428006350 & 8899520567841535 \\
\hline 0.2294557222590470 & 0.88991559568847830 & 2228015175820701 \\
\hline 0.7705442927409507 & 0.11008440431152170 & 7771985124179324 \\
\hline 0.2500121288984545 & 0.14594447812022080 & 8540600022097138 \\
\hline 0.7499878711015455 & 0.85405552187977920 & 1459399977902862 \\
\hline 0.6749815349582065 & 0.10437206229312320 & 2427796106758962 \\
\hline
\end{tabular}


$\begin{array}{llll}0.3250184650417935 & 0.8956279457068774 & 0.7572204193240992 \\ 0.1749842076111463 & 0.2427848829784907 & 0.1043677032714641 \\ 0.8250157623888512 & 0.7572151470215047 & 0.8956323047285366 \\ 0.6685980281335091 & 0.3167710896395164 & 0.1117274778032922 \\ 0.3314019718664909 & 0.6832289103604836 & 0.8882725221967078 \\ 0.1685897438053630 & 0.1117214782767846 & 0.3167522928958562 \\ 0.8314102411946394 & 0.8882785217232154 & 0.6832477071041438 \\ 0.2810109635852029 & 0.6652931314884256 & 0.1477733469800242 \\ 0.7189890064147946 & 0.3347068685115744 & 0.8522266380199710 \\ 0.2189939313219540 & 0.8522045761656045 & 0.3346786509641930 \\ 0.7810060536780412 & 0.1477954238343955 & 0.6653213490358070\end{array}$

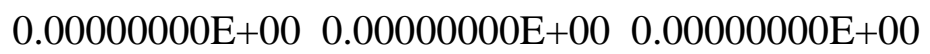

$\begin{array}{lllll}0.00000000 \mathrm{E}+00 & 0.00000000 \mathrm{E}+00 \quad 0.00000000 \mathrm{E}+00\end{array}$

$\begin{array}{lllll}0.00000000 \mathrm{E}+00 & 0.00000000 \mathrm{E}+00 \quad 0.00000000 \mathrm{E}+00\end{array}$

$\begin{array}{llll}0.00000000 \mathrm{E}+00 \quad 0.00000000 \mathrm{E}+00 \quad 0.00000000 \mathrm{E}+00 & 0.000000 \mathrm{E}+00\end{array}$

$0.00000000 \mathrm{E}+00 \quad 0.00000000 \mathrm{E}+00 \quad 0.00000000 \mathrm{E}+00$

$\begin{array}{lllll}0.00000000 \mathrm{E}+00 & 0.00000000 \mathrm{E}+00 \quad 0.00000000 \mathrm{E}+00\end{array}$

$\begin{array}{lllll}0.00000000 \mathrm{E}+00 & 0.00000000 \mathrm{E}+00 \quad 0.00000000 \mathrm{E}+00\end{array}$

$\begin{array}{lllll}0.00000000 \mathrm{E}+00 & 0.00000000 \mathrm{E}+00 \quad 0.00000000 \mathrm{E}+00\end{array}$

$0.00000000 \mathrm{E}+00 \quad 0.00000000 \mathrm{E}+00 \quad 0.00000000 \mathrm{E}+00$

$\begin{array}{llll}0.00000000 \mathrm{E}+00 \quad 0.00000000 \mathrm{E}+00 \quad 0.00000000 \mathrm{E}+00 & 0.000000 \mathrm{E}+00\end{array}$

$\begin{array}{lllll}0.00000000 \mathrm{E}+00 & 0.00000000 \mathrm{E}+00 \quad 0.00000000 \mathrm{E}+00\end{array}$

$\begin{array}{lllll}0.00000000 \mathrm{E}+00 & 0.00000000 \mathrm{E}+00 \quad 0.00000000 \mathrm{E}+00\end{array}$

$\begin{array}{llll}0.00000000 \mathrm{E}+00 \quad 0.00000000 \mathrm{E}+00 \quad 0.00000000 \mathrm{E}+00 & 0.000000 \mathrm{E}+00\end{array}$

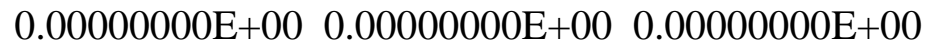

$\begin{array}{llll}0.00000000 \mathrm{E}+00 \quad 0.00000000 \mathrm{E}+00 \quad 0.00000000 \mathrm{E}+00 & 0.000000 \mathrm{E}+00\end{array}$

$\begin{array}{llll}0.00000000 \mathrm{E}+00 \quad 0.00000000 \mathrm{E}+00 \quad 0.00000000 \mathrm{E}+00 & 0.000000 \mathrm{E}+00\end{array}$

$\begin{array}{lllll}0.00000000 \mathrm{E}+00 & 0.00000000 \mathrm{E}+00 & 0.00000000 \mathrm{E}+00\end{array}$

$\begin{array}{llll}0.00000000 \mathrm{E}+00 & 0.00000000 \mathrm{E}+00 \quad 0.00000000 \mathrm{E}+00\end{array}$

$\begin{array}{lllll}0.00000000 \mathrm{E}+00 & 0.00000000 \mathrm{E}+00 \quad 0.00000000 \mathrm{E}+00\end{array}$

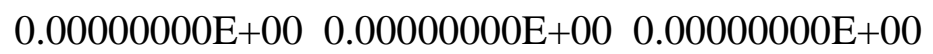

$\begin{array}{lllll}0.00000000 \mathrm{E}+00 & 0.00000000 \mathrm{E}+00 \quad 0.00000000 \mathrm{E}+00\end{array}$

$\begin{array}{lllll}0.00000000 \mathrm{E}+00 & 0.00000000 \mathrm{E}+00 & 0.00000000 \mathrm{E}+00\end{array}$

$0.00000000 \mathrm{E}+00 \quad 0.00000000 \mathrm{E}+00 \quad 0.00000000 \mathrm{E}+00$

$\begin{array}{lllll}0.00000000 \mathrm{E}+00 & 0.00000000 \mathrm{E}+00 \quad 0.00000000 \mathrm{E}+00\end{array}$

$\begin{array}{lllll}0.00000000 \mathrm{E}+00 & 0.00000000 \mathrm{E}+00 \quad 0.00000000 \mathrm{E}+00\end{array}$

$\begin{array}{lllll}0.00000000 \mathrm{E}+00 & 0.00000000 \mathrm{E}+00 \quad 0.00000000 \mathrm{E}+00\end{array}$

$\begin{array}{lllll}0.00000000 \mathrm{E}+00 & 0.00000000 \mathrm{E}+00 \quad 0.00000000 \mathrm{E}+00\end{array}$

$0.00000000 \mathrm{E}+00 \quad 0.00000000 \mathrm{E}+00 \quad 0.00000000 \mathrm{E}+00$

$\begin{array}{lllll}0.00000000 \mathrm{E}+00 & 0.00000000 \mathrm{E}+00 \quad 0.00000000 \mathrm{E}+00\end{array}$

$\begin{array}{lllll}0.00000000 \mathrm{E}+00 & 0.00000000 \mathrm{E}+00 \quad 0.00000000 \mathrm{E}+00\end{array}$

$\begin{array}{lllll}0.00000000 \mathrm{E}+00 & 0.00000000 \mathrm{E}+00 \quad 0.00000000 \mathrm{E}+00\end{array}$

$\begin{array}{lllll}0.00000000 \mathrm{E}+00 & 0.00000000 \mathrm{E}+00 \quad 0.00000000 \mathrm{E}+00\end{array}$

$\begin{array}{lllll}0.00000000 \mathrm{E}+00 & 0.00000000 \mathrm{E}+00 \quad 0.00000000 \mathrm{E}+00\end{array}$

$\begin{array}{llll}0.00000000 \mathrm{E}+00 \quad 0.00000000 \mathrm{E}+00 \quad 0.00000000 \mathrm{E}+00 & 0\end{array}$ 


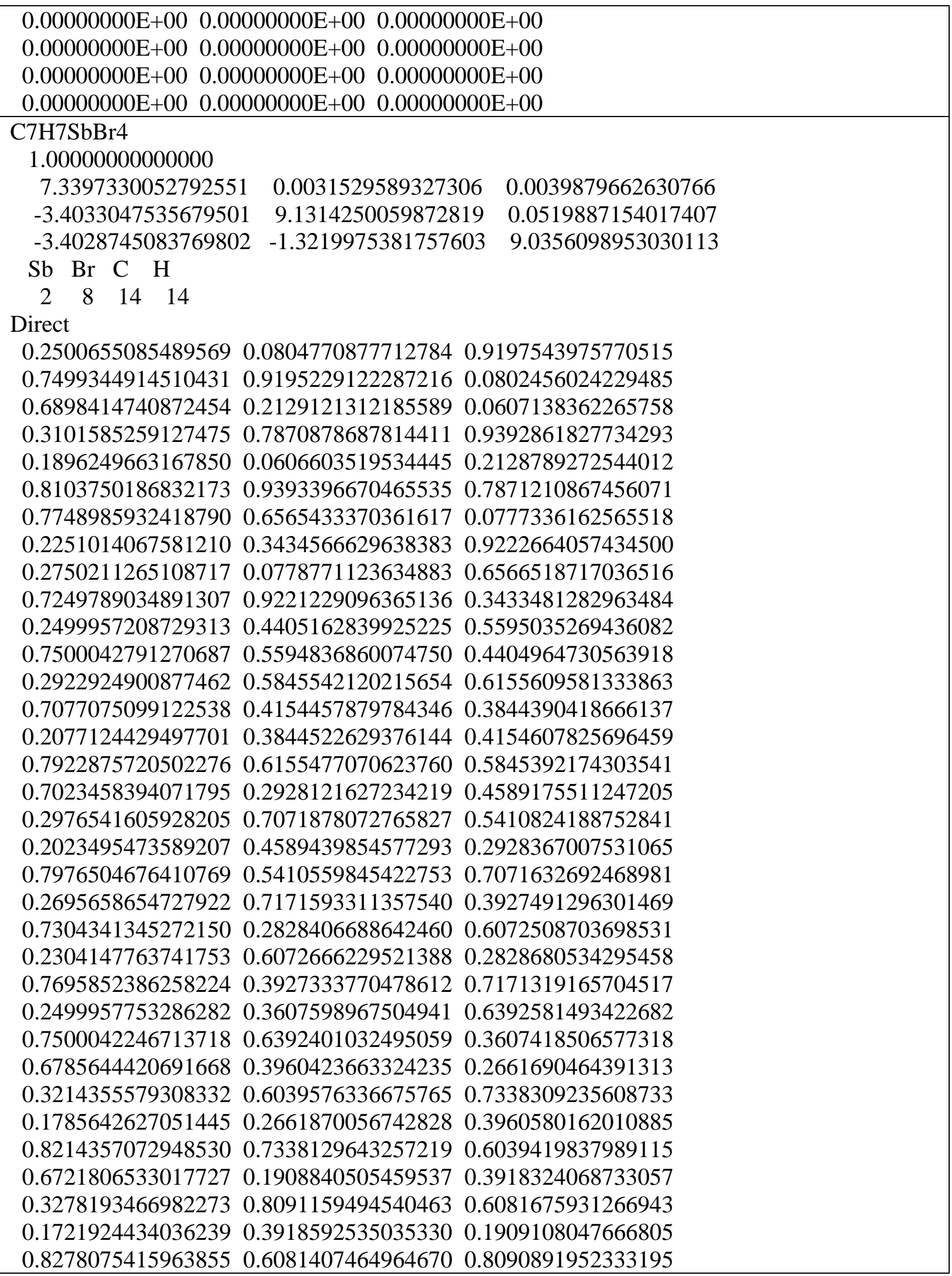




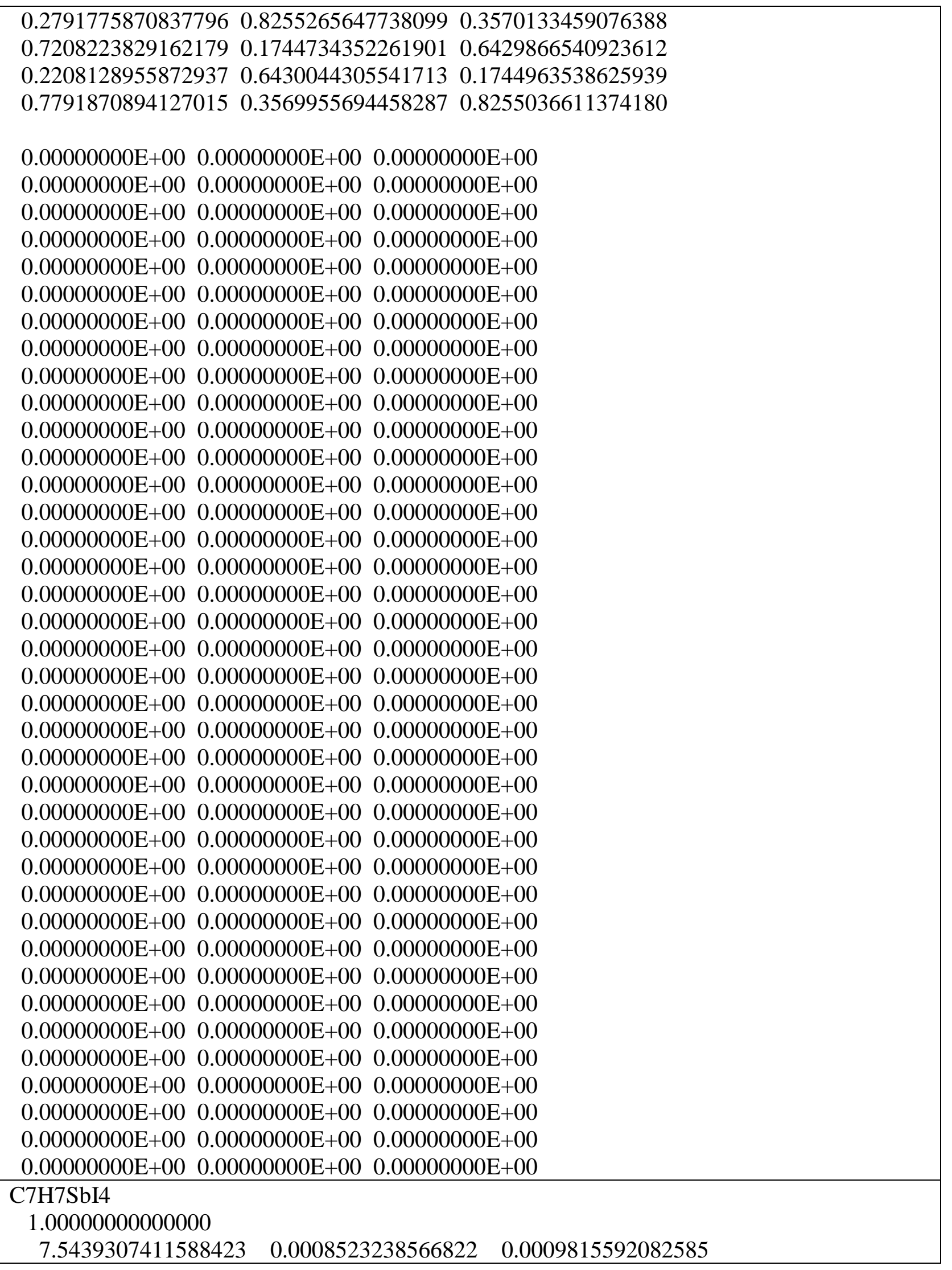




\begin{tabular}{|c|c|}
\hline-3.2943920171823873 & $\begin{array}{lll}9 & 9.6624281204033640 & 0.0461614316217994\end{array}$ \\
\hline-3.2943920171825214 & -1.3094047258020010 \\
\hline $\mathrm{Sb} \quad \mathrm{I} \quad \mathrm{C} \quad \mathrm{H}$ & \\
\hline $\begin{array}{llll}2 & 8 & 14 & 14\end{array}$ & \\
\hline Direct & \\
\hline 0.2500000000000000 & $\begin{array}{lll}0.0822164954900160 & 0.9177834825099822\end{array}$ \\
\hline 0.7500000000000000 & 0.91778348250998220 .0822165174900178 \\
\hline 0.6970545981685277 & 0.21241073200206270 .0615146447701989 \\
\hline 0.3029454018314723 & $0.7875892829979350 \quad 0.9384853472298005$ \\
\hline 0.1970545981685277 & 0.06151464477019890 .2124107320020627 \\
\hline 0.8029454018314723 & 0.93848534722980050 .7875892829979350 \\
\hline 0.2165000059812741 & $0.3548082021276997 \quad 0.9197328563837175$ \\
\hline 0.7834999790187283 & 0.64519179787230030 .0802671436162825 \\
\hline 0.2835000090187165 & $0.0802671366162855 \quad 0.6451917978723003$ \\
\hline 0.7165000209812717 & 0.91973285638371750 .3548082021276997 \\
\hline 0.2500000000000000 & 0.44324752542598840 .5567525045740211 \\
\hline 0.7500000000000000 & $\begin{array}{lll}0.5567524445740091 & 0.4432474954259789\end{array}$ \\
\hline 0.2893514095681411 & 0.58003919562604980 .6101531554890087 \\
\hline 0.7106485904318589 & 0.41996080437395020 .3898468445109913 \\
\hline 0.2106486054318637 & 0.38984687451102220 .4199608343739740 \\
\hline 0.7893514095681340 & $0.6101531554889803 \quad 0.5800391956260285$ \\
\hline 0.7055336995804495 & $0.3034820973141166 \quad 0.4602456190632864$ \\
\hline $0.2944663004195505 \mathrm{c}$ & $\begin{array}{lll}0.6965179026858834 & 0.5397543809367136\end{array}$ \\
\hline $0.2055336995804495 \mathrm{c}$ & $0.4602456190633148 \quad 0.3034820973141024$ \\
\hline $0.7944663004195505 \mathrm{c}$ & 0.53975438093668520 .6965179026858976 \\
\hline $0.2680413823807157 \mathrm{C}$ & $0.7058267850737678 \quad 0.3989220130266986$ \\
\hline $0.7319586176192843 \mathrm{C}$ & 0.29417321492623220 .6010779869733014 \\
\hline $0.2319586176192701 \mathrm{c}$ & $\begin{array}{lll}0.6010779869733085 & 0.2941731849261799\end{array}$ \\
\hline 0.7680413823807299 & $0.3989220130266915 \quad 0.7058267850738176$ \\
\hline 0.2499999999999929 c & 0.36753822104936520 .6324617789505922 \\
\hline $0.7500000000000071 \mathrm{c}$ & $0.6324617789506348 \quad 0.3675382210494078$ \\
\hline 0.6832914498172897 c & 0.40200526013129690 .2774546017674311 \\
\hline $0.3167085501827103 \mathrm{C}$ & $\begin{array}{lll}0.5979947398687031 & 0.7225453682325735\end{array}$ \\
\hline $0.1832914198172801 \mathrm{C}$ & 0.27745460176737420 .4020052601312969 \\
\hline $0.8167085501827174 \mathrm{C}$ & $0.7225453682326304 \quad 0.5979947398687031$ \\
\hline $0.6774096882158318 \mathrm{c}$ & $0.2062354278815803 \quad 0.3965416244730804$ \\
\hline $0.3225903117841682 \mathrm{c}$ & $0.7937645721184197 \quad 0.6034583755269196$ \\
\hline $0.1774096732158341 \mathrm{c}$ & $0.3965416244730520 \quad 0.2062354278816301$ \\
\hline $0.8225903117841682 \mathrm{c}$ & $\begin{array}{lll}0.6034583755269480 & 0.7937645721183699\end{array}$ \\
\hline $0.2760962790666639 \mathrm{c}$ & $0.8086073409384653 \quad 0.3651220526155399$ \\
\hline $0.7239036909333336 \mathrm{c}$ & $\begin{array}{lll}0.1913926590615347 & 0.6348779473844601\end{array}$ \\
\hline $0.2239037359333409 \mathrm{c}$ & 0.63487794738447430 .1913926740615821 \\
\hline $0.7760962490666543 \mathrm{c}$ & $0.3651220526155257 \quad 0.8086073409384227$ \\
\hline $0.00000000 \mathrm{E}+00 \quad 0.000$ & $000000 \mathrm{E}+00 \quad 0.00000000 \mathrm{E}+00$ \\
\hline $0.00000000 \mathrm{E}+00 \quad 0.000$ & $000000 \mathrm{E}+00 \quad 0.00000000 \mathrm{E}+00$ \\
\hline
\end{tabular}




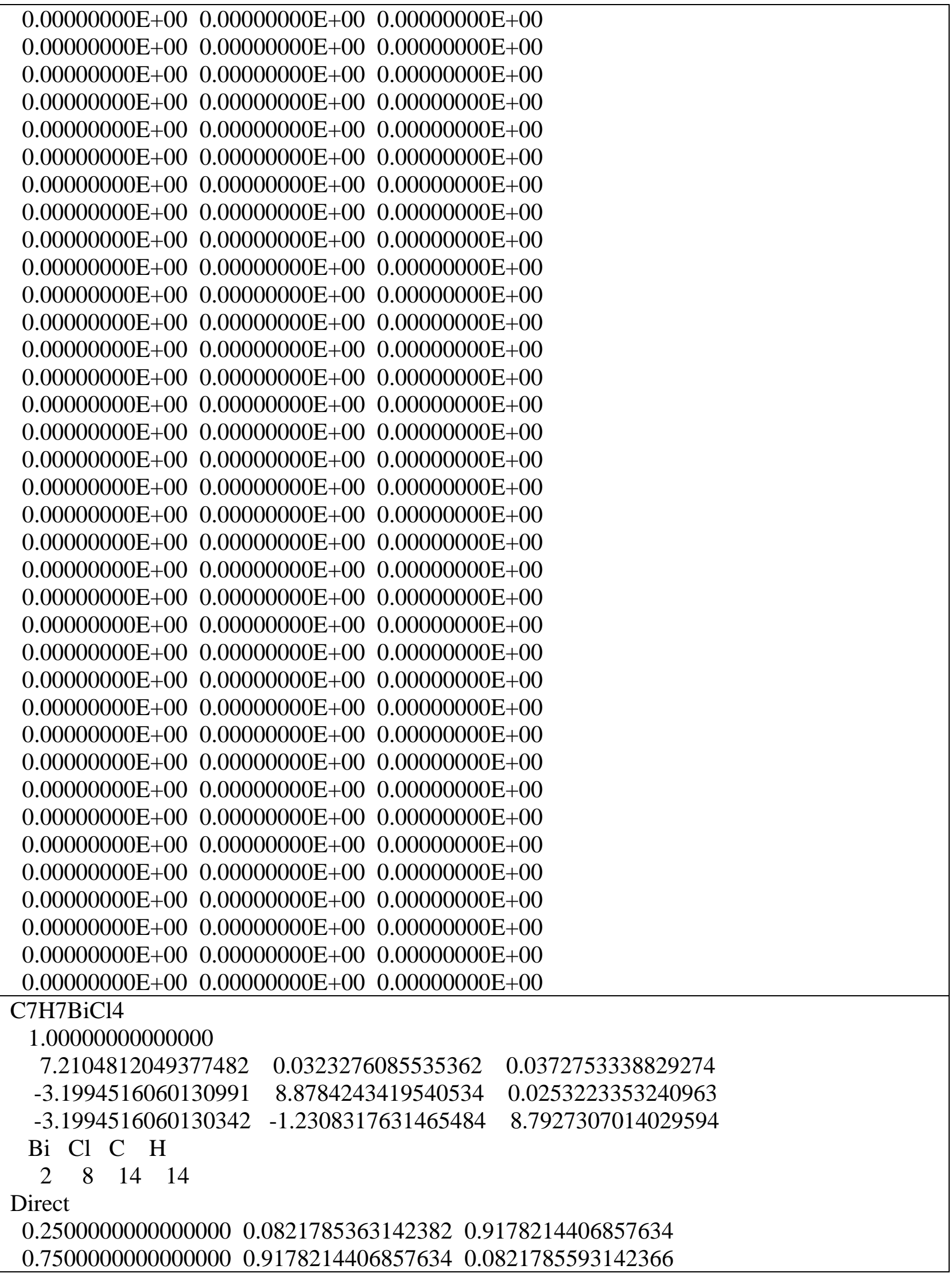


$\begin{array}{lllll}0.6791945500334364 & 0.2042961103272631 & 0.0576871784503936\end{array}$ $\begin{array}{lllll}0.3208054499665636 & 0.7957039196727322 & 0.9423128215496064\end{array}$ $\begin{array}{lllll}0.1791945650334412 & 0.0576871784503936 & 0.2042961103272631\end{array}$ $\begin{array}{lllll}0.8208054499665636 & 0.9423128215496064 & 0.7957039196727322\end{array}$ $\begin{array}{lllll}0.2243501707184166 & 0.3460549971896896 & 0.9215595657740394\end{array}$ $\begin{array}{lllll}0.7756498152815823 & 0.6539450328103129 & 0.0784404342259606\end{array}$ $\begin{array}{lllll}0.2756498152815823 & 0.0784404562259624 & 0.6539450328103129\end{array}$ $\begin{array}{lllll}0.7243501847184177 & 0.9215595657740394 & 0.3460549671896871\end{array}$ $\begin{array}{llll}0.2500000000000000 & 0.4379534532316498 & 0.5620465167683477\end{array}$ $\begin{array}{lllll}0.7500000000000000 & 0.5620465767683527 & 0.4379534832316523\end{array}$ $\begin{array}{lllll}0.2876048271070246 & 0.5854700421724246 & 0.6190564430001331\end{array}$ $\begin{array}{llllll}0.7123951428929729 & 0.4145299578275754 & 0.3809435569998669\end{array}$ $\begin{array}{llllll}0.2123951728929754 & 0.3809435269998644 & 0.4145299578275754\end{array}$ $\begin{array}{lllll}0.7876048571070271 & 0.6190565020001344 & 0.5854700421724246\end{array}$ $\begin{array}{lllll}0.7075885883337563 & 0.2882657632502870 & 0.4579051040744204\end{array}$ $\begin{array}{lllll}0.2924114116662437 & 0.7117342667497084 & 0.5420948959255796\end{array}$ $\begin{array}{lllll}0.2075885733337586 & 0.4579051040744204 & 0.2882657632502870\end{array}$ $\begin{array}{lllll}0.7924114116662437 & 0.5420948959255796 & 0.7117342667497084\end{array}$ $\begin{array}{lllll}0.2671096024139317 & 0.7224564252852304 & 0.3898932073585257\end{array}$ $\begin{array}{lllll}0.7328903975860683 & 0.2775435747147696 & 0.6101067626414718\end{array}$ $\begin{array}{lllll}0.2328903975860683 & 0.6101068226414696 & 0.2775435747147696\end{array}$ $\begin{array}{lllll}0.7671096024139317 & 0.3898931773585304 & 0.7224564252852304\end{array}$ $\begin{array}{lllll}0.2500000000000000 & 0.3558166114280965 & 0.6441833595719046\end{array}$ $\begin{array}{lllll}0.7500000000000000 & 0.6441833595719046 & 0.3558166404280954\end{array}$ $\begin{array}{lllll}0.6862014414068440 & 0.3949522758355499 & 0.2597070056598554\end{array}$ $\begin{array}{lllll}0.3137985585931560 & 0.6050476941644547 & 0.7402929943401446\end{array}$ $\begin{array}{lllll}0.1862014114068415 & 0.2597070056598554 & 0.3949522758355499\end{array}$ $\begin{array}{llll}0.8137985585931560 & 0.7402929943401446 & 0.6050476941644547\end{array}$ $\begin{array}{lllll}0.6811371682771465 & 0.1834933102185730 & 0.3901598225142138\end{array}$ $\begin{array}{lllll}0.3188628317228535 & 0.8165066747814294 & 0.6098401474857909\end{array}$ $\begin{array}{lllll}0.1811371532771489 & 0.3901598225142138 & 0.1834933102185730\end{array}$ $\begin{array}{lllll}0.8188628317228535 & 0.6098401474857909 & 0.8165066747814294\end{array}$ $\begin{array}{lllll}0.2756012557074712 & 0.8339551137048176 & 0.3532962862198943\end{array}$ $\begin{array}{llllll}0.7243987142925263 & 0.1660448862951824 & 0.6467037137801057\end{array}$ $\begin{array}{lllll}0.2243987592925265 & 0.6467037137801057 & 0.1660448712951776\end{array}$ $\begin{array}{lllll}0.7756012257074687 & 0.3532962862198943 & 0.8339551137048176\end{array}$

$0.00000000 \mathrm{E}+00 \quad 0.00000000 \mathrm{E}+00 \quad 0.00000000 \mathrm{E}+00$ $0.00000000 \mathrm{E}+00 \quad 0.00000000 \mathrm{E}+00 \quad 0.00000000 \mathrm{E}+00$ $\begin{array}{lll}0.00000000 \mathrm{E}+00 & 0.00000000 \mathrm{E}+00 \quad 0.00000000 \mathrm{E}+00\end{array}$ $0.00000000 \mathrm{E}+00 \quad 0.00000000 \mathrm{E}+00 \quad 0.00000000 \mathrm{E}+00$ $0.00000000 \mathrm{E}+00 \quad 0.00000000 \mathrm{E}+00 \quad 0.00000000 \mathrm{E}+00$ $0.00000000 \mathrm{E}+00 \quad 0.00000000 \mathrm{E}+00 \quad 0.00000000 \mathrm{E}+00$ $0.00000000 \mathrm{E}+00 \quad 0.00000000 \mathrm{E}+00 \quad 0.00000000 \mathrm{E}+00$ $0.00000000 \mathrm{E}+00 \quad 0.00000000 \mathrm{E}+00 \quad 0.00000000 \mathrm{E}+00$ $0.00000000 \mathrm{E}+00 \quad 0.00000000 \mathrm{E}+00 \quad 0.00000000 \mathrm{E}+00$ 


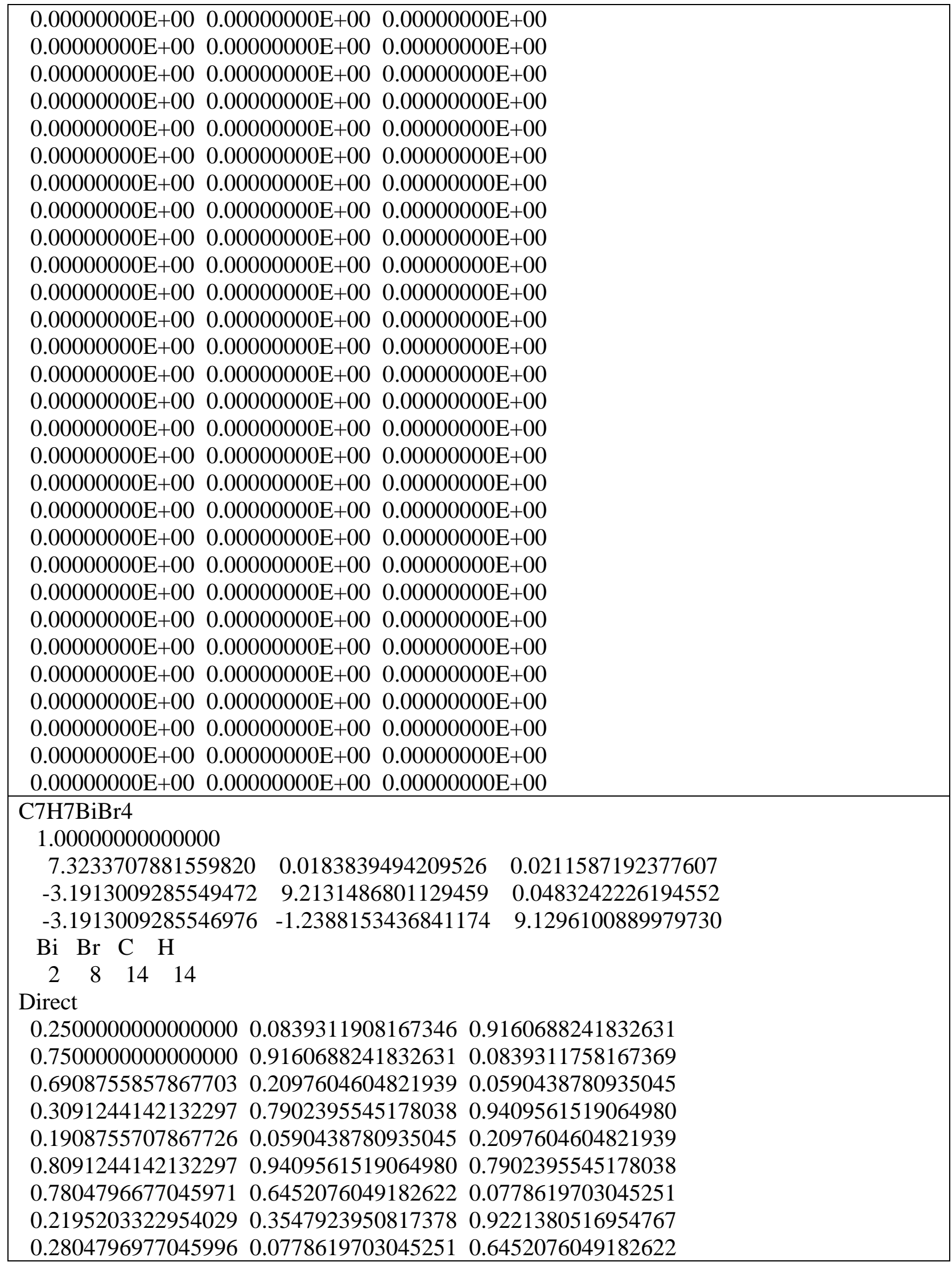




\begin{tabular}{|c|}
\hline $\begin{array}{llll}0.7195202722954051 & 0.9221380516954767 & 0.3547923950817378\end{array}$ \\
\hline $\begin{array}{llll}0.2500000000000000 & 0.4380887655055261 & 0.5619112644944835\end{array}$ \\
\hline $\begin{array}{llll}0.7500000000000000 & 0.5619112044944714 & 0.4380887355055165\end{array}$ \\
\hline $\begin{array}{llll}0.2907971872337072 & 0.5811313544193553 & 0.6177102688547791\end{array}$ \\
\hline $\begin{array}{llll}0.7092028127662928 & 0.4188686455806447 & 0.3822897311452209\end{array}$ \\
\hline $\begin{array}{llll}0.2092028277662834 & 0.3822897611452234 & 0.4188686755806330\end{array}$ \\
\hline $\begin{array}{llll}0.7907971872337143 & 0.6177102688547791 & 0.5811313544193695\end{array}$ \\
\hline $\begin{array}{llll}0.7031856839443691 & 0.2965672770182337 & 0.4562727058474536\end{array}$ \\
\hline $\begin{array}{llll}0.2968143160556309 & 0.7034327229817663 & 0.5437272941525464\end{array}$ \\
\hline $\begin{array}{llll}0.2031856839443691 & 0.4562727058474394 & 0.2965672770182337\end{array}$ \\
\hline $\begin{array}{lll}0.7968143160556309 & 0.5437272941525606 & 0.7034327229817663\end{array}$ \\
\hline $\begin{array}{llll}0.2693266941358488 & 0.7134145613861449 & 0.3961828240665568\end{array}$ \\
\hline $\begin{array}{llll}0.7306733058641512 & 0.2865854386138551 & 0.6038171759334432\end{array}$ \\
\hline $\begin{array}{llll}0.2306733058641512 & 0.6038171759334361 & 0.2865854086138739\end{array}$ \\
\hline $\begin{array}{llll}0.7693266941358488 & 0.3961828240665639 & 0.7134145613861236\end{array}$ \\
\hline $\begin{array}{llll}0.2500000000000071 & 0.3586473131365935 & 0.6413526868634420\end{array}$ \\
\hline $\begin{array}{lll}0.7499999999999929 & 0.6413526868634065 & 0.3586473131365580\end{array}$ \\
\hline $\begin{array}{llll}0.6812586251852366 & 0.4003692273596329 & 0.2646234955614091\end{array}$ \\
\hline $\begin{array}{llll}0.3187413748147634 & 0.5996307726403671 & 0.7353764744385956\end{array}$ \\
\hline $\begin{array}{llll}0.1812585951852412 & 0.2646234955614588 & 0.4003692273596329\end{array}$ \\
\hline $\begin{array}{lll}0.8187413748147563 & 0.7353764744385458 & 0.5996307726403671\end{array}$ \\
\hline $\begin{array}{llll}0.6732816142045195 & 0.1947886119681499 & 0.3896749729551985\end{array}$ \\
\hline $\begin{array}{llll}0.3267183857954805 & 0.8052113880318501 & 0.6103250270448015\end{array}$ \\
\hline $\begin{array}{llll}0.1732815992045147 & 0.3896749729552269 & 0.1947886119681144\end{array}$ \\
\hline $\begin{array}{llll}0.8267183857954876 & 0.6103250270447731 & 0.8052113880318856\end{array}$ \\
\hline $\begin{array}{llll}0.2792330850061049 & 0.8214831191273433 & 0.3609583760354624\end{array}$ \\
\hline $\begin{array}{llll}0.7207668849938926 & 0.1785168808726567 & 0.6390416239645376\end{array}$ \\
\hline $\begin{array}{llll}0.2207669299938786 & 0.6390416239645234 & 0.1785168958726189\end{array}$ \\
\hline $\begin{array}{llll}0.7792330550061166 & 0.3609583760354766 & 0.8214831191273859\end{array}$ \\
\hline $0.00000000 \mathrm{E}+00 \quad 0.00000000 \mathrm{E}+00 \quad 0.00000000 \mathrm{E}+00$ \\
\hline $0.00000000 \mathrm{E}+00 \quad 0.00000000 \mathrm{E}+00 \quad 0.00000000 \mathrm{E}+00$ \\
\hline $0.00000000 \mathrm{E}+00 \quad 0.00000000 \mathrm{E}+00 \quad 0.00000000 \mathrm{E}+00$ \\
\hline $0.00000000 \mathrm{E}+00 \quad 0.00000000 \mathrm{E}+00 \quad 0.00000000 \mathrm{E}+00$ \\
\hline $0.00000000 \mathrm{E}+00 \quad 0.00000000 \mathrm{E}+00 \quad 0.00000000 \mathrm{E}+00$ \\
\hline $0.00000000 \mathrm{E}+00 \quad 0.00000000 \mathrm{E}+00 \quad 0.00000000 \mathrm{E}+00$ \\
\hline $0.00000000 \mathrm{E}+00 \quad 0.00000000 \mathrm{E}+00 \quad 0.00000000 \mathrm{E}+00$ \\
\hline $0.00000000 \mathrm{E}+00 \quad 0.00000000 \mathrm{E}+00 \quad 0.00000000 \mathrm{E}+00$ \\
\hline $0.00000000 \mathrm{E}+00 \quad 0.00000000 \mathrm{E}+00 \quad 0.00000000 \mathrm{E}+00$ \\
\hline $0.00000000 \mathrm{E}+00 \quad 0.00000000 \mathrm{E}+00 \quad 0.00000000 \mathrm{E}+00$ \\
\hline $0.00000000 \mathrm{E}+00 \quad 0.00000000 \mathrm{E}+00 \quad 0.00000000 \mathrm{E}+00$ \\
\hline $0.00000000 \mathrm{E}+00 \quad 0.00000000 \mathrm{E}+00 \quad 0.00000000 \mathrm{E}+00$ \\
\hline $0.00000000 \mathrm{E}+00 \quad 0.00000000 \mathrm{E}+00 \quad 0.00000000 \mathrm{E}+00$ \\
\hline $0.00000000 \mathrm{E}+00 \quad 0.00000000 \mathrm{E}+00 \quad 0.00000000 \mathrm{E}+00$ \\
\hline $0.00000000 \mathrm{E}+00 \quad 0.00000000 \mathrm{E}+00 \quad 0.00000000 \mathrm{E}+00$ \\
\hline $0.00000000 \mathrm{E}+00 \quad 0.00000000 \mathrm{E}+00 \quad 0.00000000 \mathrm{E}+00$ \\
\hline
\end{tabular}




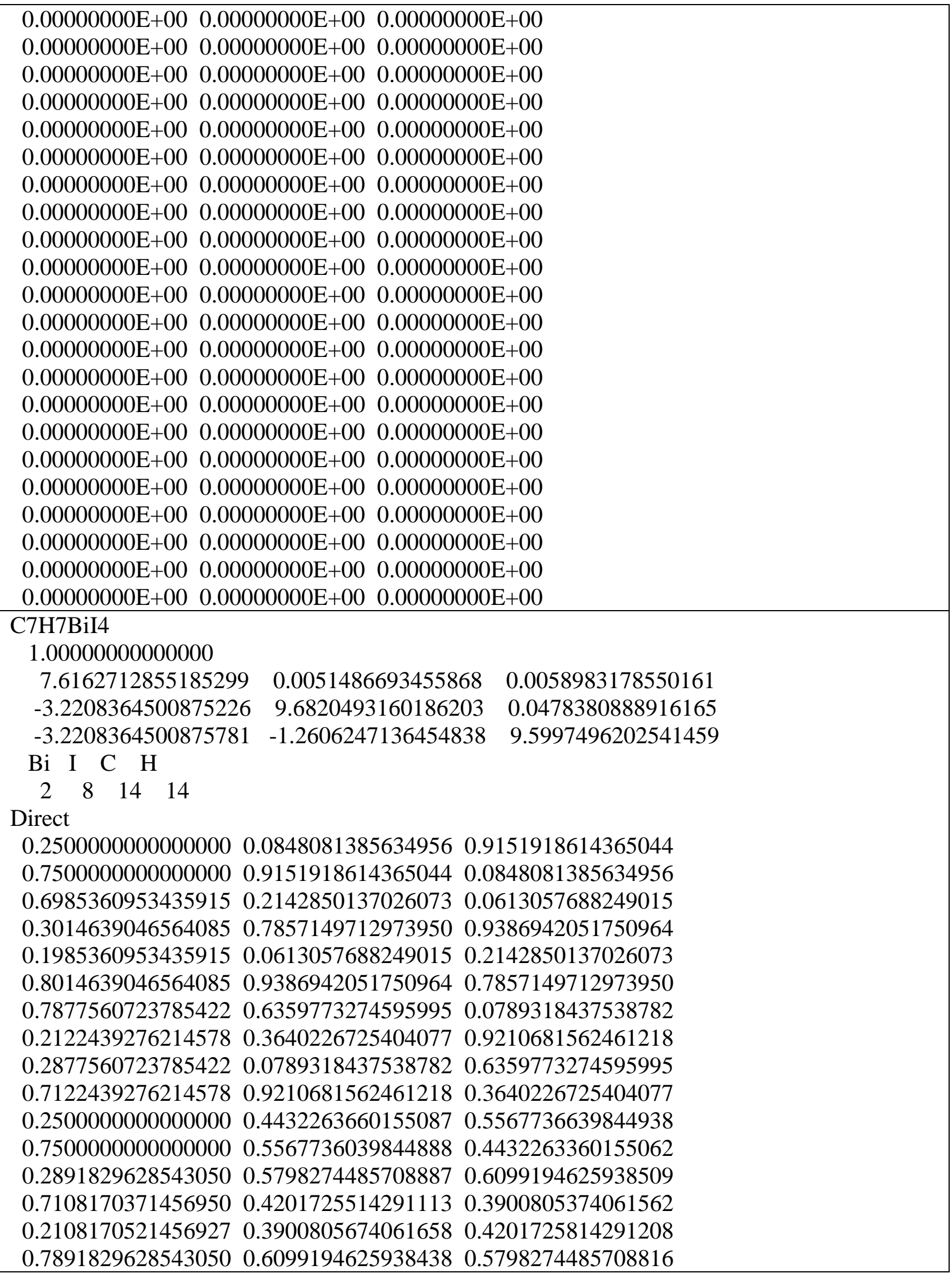


$\begin{array}{lllll}0.7051093394396304 & 0.3035801326273386 & 0.4605872464591059\end{array}$ $\begin{array}{lllll}0.2948906605603696 & 0.6964198673726614 & 0.5394127535408941\end{array}$ $\begin{array}{lllll}0.2051093394396304 & 0.4605872464591059 & 0.3035801326273386\end{array}$ $\begin{array}{lllll}0.7948906605603696 & 0.5394127535408941 & 0.6964198673726614\end{array}$ $\begin{array}{lllll}0.2685139962583847 & 0.7061065739316490 & 0.3987372342961919\end{array}$ $\begin{array}{lllll}0.7314860037416153 & 0.2938934260683510 & 0.6012627657038081\end{array}$ $\begin{array}{llllll}0.2314860037416153 & 0.6012627657038081 & 0.2938933960683414\end{array}$ $\begin{array}{lllll}0.7685139962583847 & 0.3987372342961919 & 0.7061065739316561\end{array}$ $\begin{array}{lllll}0.2500000000000000 & 0.3674470622892372 & 0.6325529377107557\end{array}$ $\begin{array}{lllll}0.7500000000000000 & 0.6325529377107628 & 0.3674470622892443\end{array}$ $\begin{array}{lllll}0.6843520408752966 & 0.4027035986655250 & 0.2778604307076833\end{array}$ $\begin{array}{lllll}0.3156479591247034 & 0.5972964013344750 & 0.7221395392923213\end{array}$ $\begin{array}{lllll}0.1843520108752941 & 0.2778604307076762 & 0.4027035986655250\end{array}$ $\begin{array}{lllll}0.8156479591247034 & 0.7221395392923284 & 0.5972964013344750\end{array}$ $\begin{array}{llllll}0.6764892478971234 & 0.2063070090599624 & 0.3969851480734405\end{array}$ $\begin{array}{llll}0.3235107521028766 & 0.7936929909400376 & 0.6030148519265595\end{array}$ $\begin{array}{lllll}0.1764892328971257 & 0.3969851480734334 & 0.2063070090599695\end{array}$ $\begin{array}{lllll}0.8235107521028766 & 0.6030148519265666 & 0.7936929909400305\end{array}$ $\begin{array}{lllll}0.2768188698811755 & 0.8089950297443522 & 0.3651421865227533\end{array}$ $\begin{array}{lllll}0.7231811001188220 & 0.1910049702556478 & 0.6348578134772467\end{array}$ $\begin{array}{lllll}0.2231811451188221 & 0.6348578134772538 & 0.1910049852556668\end{array}$ $\begin{array}{lllll}0.7768188398811660 & 0.3651421865227462 & 0.8089950297443380\end{array}$

$0.00000000 \mathrm{E}+00 \quad 0.00000000 \mathrm{E}+00 \quad 0.00000000 \mathrm{E}+00$ $\begin{array}{lll}0.00000000 \mathrm{E}+00 & 0.00000000 \mathrm{E}+00 & 0.00000000 \mathrm{E}+00\end{array}$ $\begin{array}{llll}0.00000000 \mathrm{E}+00 & 0.00000000 \mathrm{E}+00 \quad 0.00000000 \mathrm{E}+00\end{array}$ $\begin{array}{lll}0.00000000 \mathrm{E}+00 \quad 0.00000000 \mathrm{E}+00 & 0.00000000 \mathrm{E}+00\end{array}$ $0.00000000 \mathrm{E}+00 \quad 0.00000000 \mathrm{E}+00 \quad 0.00000000 \mathrm{E}+00$
$0.0000000 \mathrm{E}+00$ $\begin{array}{lll}0.00000000 \mathrm{E}+00 & 0.00000000 \mathrm{E}+00 \quad 0.00000000 \mathrm{E}+00\end{array}$ $\begin{array}{lll}0.00000000 \mathrm{E}+00 & 0.00000000 \mathrm{E}+00 \quad 0.00000000 \mathrm{E}+00\end{array}$

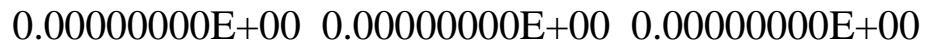

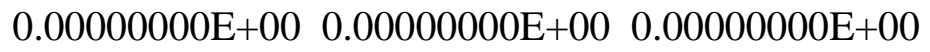
$\begin{array}{lll}0.00000000 \mathrm{E}+00 \quad 0.00000000 \mathrm{E}+00 & 0.00000000 \mathrm{E}+00\end{array}$

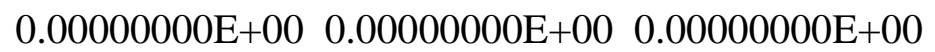

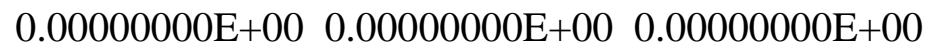

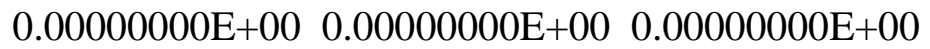

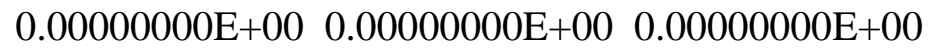

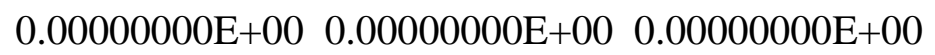

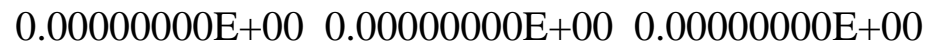

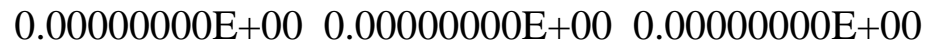

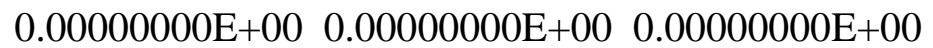
$0.00000000 \mathrm{E}+00 \quad 0.00000000 \mathrm{E}+00 \quad 0.00000000 \mathrm{E}+00$
$0.0000000 \mathrm{E}+00$

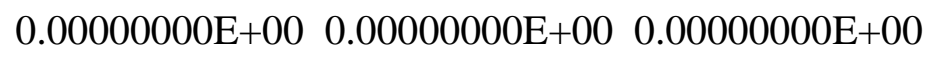

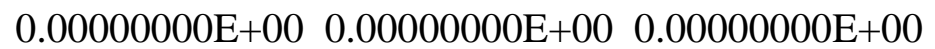

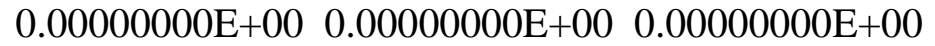

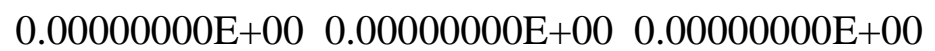




$$
\begin{array}{lll}
0.00000000 \mathrm{E}+00 & 0.00000000 \mathrm{E}+00 & 0.00000000 \mathrm{E}+00 \\
0.00000000 \mathrm{E}+00 & 0.00000000 \mathrm{E}+00 & 0.00000000 \mathrm{E}+00 \\
0.00000000 \mathrm{E}+00 & 0.00000000 \mathrm{E}+00 & 0.00000000 \mathrm{E}+00 \\
0.00000000 \mathrm{E}+00 & 0.00000000 \mathrm{E}+00 & 0.00000000 \mathrm{E}+00 \\
0.00000000 \mathrm{E}+00 & 0.00000000 \mathrm{E}+00 & 0.00000000 \mathrm{E}+00 \\
0.00000000 \mathrm{E}+00 & 0.00000000 \mathrm{E}+00 & 0.00000000 \mathrm{E}+00 \\
0.00000000 \mathrm{E}+00 & 0.00000000 \mathrm{E}+00 & 0.00000000 \mathrm{E}+00 \\
0.00000000 \mathrm{E}+00 & 0.00000000 \mathrm{E}+00 & 0.00000000 \mathrm{E}+00 \\
0.00000000 \mathrm{E}+00 & 0.00000000 \mathrm{E}+00 & 0.00000000 \mathrm{E}+00 \\
0.00000000 \mathrm{E}+00 & 0.00000000 \mathrm{E}+00 & 0.00000000 \mathrm{E}+00 \\
0.00000000 \mathrm{E}+00 & 0.00000000 \mathrm{E}+00 & 0.00000000 \mathrm{E}+00 \\
0.00000000 \mathrm{E}+00 & 0.00000000 \mathrm{E}+00 & 0.00000000 \mathrm{E}+00 \\
0.00000000 \mathrm{E}+00 & 0.00000000 \mathrm{E}+00 & 0.00000000 \mathrm{E}+00 \\
0.00000000 \mathrm{E}+00 & 0.00000000 \mathrm{E}+00 & 0.00000000 \mathrm{E}+00 \\
0.00000000 \mathrm{E}+00 & 0.00000000 \mathrm{E}+00 & 0.00000000 \mathrm{E}+00
\end{array}
$$

